\title{
Analysis of Methods for Determining the Characteristics of a Single Spatial Electromagnetic Field
}

\author{
Abdulhak Khalikov* \\ Tashkent Institute of Railway Engineering 1001676, Tashkent, Uzbekistan
}

\begin{abstract}
This paper analyzes the methods for determining the characteristics of a single spatial electromagnetic field; considerations combine all-electric, magnetic, and electromagnetic fields into a single electromagnetic theory of a spatial field. On the basis of the concept of a single field of force spatial interaction of material bodies, it is analytically established that in a physical vacuum only waves of its polarization actually exist, transporting in the space of a vacuum sphere its excitation energy, which when force interacting with a certain physical characteristic (electrical, magnetic or gravitational) material body, creates a dynamic response of the parameters of this body, which is recorded in the experiment as a real flow of energy with responsible of the physical nature.
\end{abstract}

Keywords: Electric, gravitational, magnetic, unified spatial electromagnetic field wave equations.

\section{Introduction}

Contradictions and paradoxes in the theory began to be considered as only strange and harmless exceptions in the harmonious theory [1]. Mathematics of the theory played a significant role. The consequence of excessive mathematics of this theory turned the electromagnetic theory from the physical to the mathematical. The theory of motion in time, the energy of rotation is in many ways similar to Einstein's theory of relativity. In fact, this theory of relativity has a dual character. The first is the peak of human knowledge; the second is the theory of movement in time. In this case, it is not necessary to know this theory, since the theory of motion in time allows you to look at the theory of relativity from the new side and makes it simple and clear [2].

The theory of movement in time is an effective tool for the knowledge of nature. The theory of motion is inextricably linked with the gravitational field. The non-explosive part of electrodynamics and gravity leads to the creation of a single spatial field, which Einstein dreamed of.

The aim of the study is to combine all the fields: Electric, magnetic, electromagnetic into a single electromagnetic theory of the spatial field. With the single spatial electromagnetic field support, such phenomena as the production of fine materials that cannot be obtained by any separators, and if possible, only with a large amount of electricity, are easily explained.

\footnotetext{
*Corresponding author: Xalikov_abdulxak@mail.ru
} 
Recently, researcher pay more attentionin nanomaterials and nanotechnologies field, working with objects those dimensions are commensurate with the correlation radius of a physical phenomenon (the mean free path of electrons, photons, the coherence length in a superconductor, the dimensions of a magnetic domain of a conductor or nucleus solid phase) [3].

Based on the concept of a single field of force spatial interaction of material bodies, only waves of its polarization really exist in a physical vacuum, transporting its excitation energy in the vacuum medium space analytically and unambiguously. When it interacting with a force, has a certain physical characteristic (electrical, magnetic, gravitational) of the material body, creates a dynamic response of the parameters of this body, which is recorded in the experiment as a real flow energy of the corresponding physical nature [4].

The paradoxes of electrodynamics and the ways of the solution, as well as attempts to bring some electrodynamics positions to a single electromagnetic spatial field, are indicated $[4,5]$.

\section{Material and methods}

\subsection{Parameter description}

Analytical expressions describing different in physical nature electric, magnetic and gravitational forces are identical [6].

The formula of gravitational interaction:

$$
\bar{F}_{\text {grav }}=\gamma \frac{m_{1} \cdot m_{2}}{r^{2}} \cdot \frac{-}{r}
$$

Where is the $\mathrm{r}^{\gamma=\frac{1}{4 \pi \gamma_{0}}}$ gravitational constant.

Gravitational field strength:

$$
G_{\text {grav }}=\frac{F}{m}=\gamma \frac{m}{r^{2}}=\frac{m}{4 \pi \gamma_{0} r^{2}},
$$

The dimension of the gravitational field strength is the same as the acceleration $\mathrm{m} \mathrm{s}^{-1}$.

From (2):, $\quad \gamma_{0}=\frac{m}{4 \pi G_{\text {grav }} r^{2}}$

Dimension

$$
\left[\gamma_{0}\right]=\frac{\kappa g}{\mathrm{~m} / \mathrm{s}^{2} \cdot \mathrm{m}^{2}}=\frac{\kappa g \cdot \mathrm{s}^{2}}{\mathrm{~m}^{2}}
$$

$\gamma_{0}$ is a gravitational capacity

Electric capacity analog: $G=\frac{m}{\varphi_{\text {grav }}}$,

$\varphi_{\text {grav }}$ is the gravitational potential. 
In this regard, the dimensioncan write:

$$
\left[\gamma_{0}\right]=\left[\frac{\kappa g \cdot s^{2}}{M^{2}}\right]=\left[\frac{\kappa g}{B^{2}}\right]
$$

Mass Planck:

$$
m_{p l}=\frac{\sqrt{\hbar c}}{G_{\text {grav }}}=\sqrt{4 \pi \lambda_{0} \hbar c}=2,176 \cdot 10^{-8} \kappa g
$$

$\hbar=\frac{h}{2 \pi}$ is the modified Planck constant.

Formula (2) is presented in the form:

$$
\begin{aligned}
& F_{\text {grav }}=\frac{m_{1} m_{2}}{m_{p l}^{2}} \cdot \frac{m_{\Pi л}^{2}}{4 \pi r^{2} \gamma_{0}}=\frac{m_{1} m_{2}}{m_{p l}^{2}} \cdot \frac{4 \pi \gamma_{0} \hbar c}{4 \pi r^{2} \gamma_{0}}=\frac{m_{1} m_{2} \hbar c}{m_{p l}^{2} r^{2}} \\
& \text { Denote: }
\end{aligned}
$$

Then: $F_{\text {grav }}=A_{\text {grav }} \frac{\hbar c}{r^{2}}$.

For electric charges, as well as for gravitational:

$$
F_{\text {эл }}=\frac{q_{1} q_{2}}{q_{\text {plel }}} \cdot \frac{q_{\text {Плэл }}}{4 \pi r^{2} \varepsilon_{0}}=\frac{q_{1} q_{2}}{q_{\text {plel }}^{2}} \cdot \frac{4 \pi \varepsilon_{0} \hbar c}{4 \pi r^{2} \varepsilon_{0}}=A_{e l} \frac{\hbar c}{r^{2}},
$$

Planck's electric charge: $q_{\text {plel }}=\sqrt{4 \pi \varepsilon_{0} \hbar c}=1,875 \cdot 10^{-18} \mathrm{Kl}$ so:

$$
A_{e l}=\frac{q_{1} q_{2}}{q_{p l}^{2}} \text {. }
$$

In the interaction of magnetic charges for a magnetic field:

$$
\begin{gathered}
q_{\text {pl } \mathrm{mag}}=\sqrt{4 \pi \mu_{0} \hbar c}=7,066 \cdot 10^{-16} v b, \\
F_{\text {mag }}=\frac{q_{1} q_{2}}{q_{\text {plmag }}} \cdot \frac{q_{\text {plmag }}}{4 \pi r^{2} \mu_{0}}=\frac{q_{1 \mu} q_{2 \mu}}{q_{\text {plmag }}^{2}} \cdot \frac{4 \pi \mu_{0} \hbar c}{4 \pi r^{2} \mu_{0}}=A_{\text {mag }} \frac{\hbar c}{r^{2}} .
\end{gathered}
$$

\subsection{The parameter theory}

Based on Formulas $(7,8,10)$ represent the equations of a single spatial electromagnetic interaction. These equations indicate the force interaction of material bodies.

The electromagnetic field is characterized by four-vectors $\bar{E}, \bar{D}, \bar{B}, \bar{H}$. For environments with constant electrical and magnetic permeability:

$$
\begin{gathered}
\bar{D}=\varepsilon \varepsilon_{0} \bar{E}, \\
\bar{B}=\mu \mu_{0} \bar{H},
\end{gathered}
$$


Therefore, when calculating, it suffices to determine only two vectors.

Usually determined from $\bar{E}$ and $\bar{H}$ the Maxwell equation.

$$
\begin{aligned}
& \operatorname{rot} \bar{H}=\gamma \bar{E}+\varepsilon \varepsilon_{0} \frac{\partial \bar{E}}{\partial t}, \\
& \operatorname{rot} \bar{E}=-\mu \mu_{0} \frac{\partial \bar{H}}{\partial t}
\end{aligned}
$$

For the uniqueness of definition $\bar{E}$ and $\bar{H}$, these equations are not enough.

Therefore, the divergence must also be known $\bar{E}$ and $\bar{H}$. By the Gauss theorem in the differential Formula(7-10):

$\operatorname{div} \bar{D}=\rho$

With a constant value $\varepsilon$ : $\operatorname{div} \bar{E}=\frac{\rho}{\varepsilon \varepsilon_{0}}$.

The main property of the magnetic field is its solenoidality:

$$
\operatorname{div} \bar{B}=0
$$

By equation (17) can be determined:

$$
\operatorname{div} \bar{H}=0 \text {. }
$$

At the interface of two media values if $\varepsilon, \mu, \gamma$ vary in jumps; therefore, a discontinuity of the field vector continuity will take place on the interface, and the boundary conditions obtained for constant fields of time remain valid for the instantaneous values of the projections of the electromagnetic field vectors.

It is possible to show the basis of the primary basic laws of electromagnetism-Coulomb's law of the interaction of fixed electric point charges:

$$
\bar{F}=\frac{q_{1} q_{2}}{4 \pi \varepsilon \varepsilon_{0} r^{2}} 1_{r}
$$

and the law of conservation of electric charge:

$$
\operatorname{div} \bar{\sigma}+\frac{\partial \rho}{\partial t}=0
$$

a chain of consecutive physical and mathematical arguments can be used to construct a system of Maxwell's electrodynamics' equations.

The functionality of the Coulomb's law consists in the fact that it describes the power interaction of stationary charged material bodies spaced apart in space. The consequence of this interaction is tension: 


$$
\bar{E}=\frac{\bar{F}}{q_{0}},(21) \quad q_{0} \text { is the trial point charge. }
$$

Because $\bar{E}$ inversely proportional $r^{2}$, then the integral of the function over a sphere

of any radius is constant:

$$
r^{2}\left(\frac{1}{r^{2}}\right) \cdot 4 \pi r^{2}=4 \pi
$$

and when using the solid angle, the flux of the vector of electric induction field $\bar{D}=\varepsilon \varepsilon_{0} \bar{E}$ through an arbitrary closed surface $\mathrm{S}$, which is identically equal to the total external charge q in volume $V_{S}$, covered by this surface

$$
\Phi_{e}=\phi_{S}, \quad D d S=\int_{v s} \rho d V=q
$$

This is the Gauss theorem. If there is an external charge, it does not mean that there is no electric field, since the charges are positive and negative, and the indicated field can be generated by neutral electric sources (electric dipoles). The property of the electric field distinguishes it from the field of Newton, in which the source of the field is gravitational masses, having only one sign - positive [11].

In the system of electrodynamics differential equations, the Gauss theorem in differential form describes the result of the electric polarization of the medium, where with electrical neutrality $(\rho=0)$ has the appearance $\operatorname{div} \bar{D}=0$. Based on equation (21), one can derive the continuity equation.

The law of conservation of charge: The change in charge at a given point in space $\frac{\partial \rho}{\partial t}$

only possible due to the movement of charge from the outside

$\operatorname{div} \bar{\sigma}$.

Divergence is the volume density of the flux of a vector field at a given point

Then:

$$
\operatorname{div}\left(\bar{\sigma}+\frac{\partial \bar{D}}{\partial t}\right)=0
$$

Given that $\operatorname{divrot} \bar{A}=0$ we will receive:

$$
\operatorname{rot} \bar{H}=\bar{\sigma}+\frac{\partial \bar{D}}{\partial t}
$$

This is the law of total current: Electrical conduction and displacement currents generate an eddy magnetic field, the strength lines of which intensity vectors span the lines of these currents.

In the field of implementation of moving charges and time-varying electric fields rot $\bar{H}(r) \neq 0$ function $\bar{H}(r)$ is purely vortex [12].

According to the definition of divergence, one can derive an equation, $\operatorname{div} \bar{B}=0$. 
Herefore, equation (17) describes not only the vortex properties $\bar{H}(r)$ but its potential version with no field sources $\operatorname{rot} \bar{H}(r)=0$.These equations are the result of medium polarization.

The electromagnetic field is not a field in the form adequate for this concept. There is a dynamic interaction between electric and magnetic fields. Such interaction can only be dynamic, a change in one is accompanied by a change in the other. Rotating, for example, two circles in two mutually perpendicular directions and a sinusoid itself, it turns out, hence two types of tension - statistical, according to the expanding sphere and dynamic, according to harmonic oscillation.

The common gravitational, electric, magnetic fields and electromagnetic waves is the environment in which they form and propagate. The Spatio-temporal environment, manifesting physical properties, behaves like any other elastic medium; it does not manifest itself as it does until it is affected. When it is affected, it responds adequately - it forms the fields of the corresponding intensity, there by fulfilling the role of the working medium for transmitting the impact [13].

Based on the concept of a unified field of force spatial interaction of material bodies [14], it was analytically established that in a physical vacuum only waves of its polarization actually exist and transfer its excitation energy in the space of a vacuum sphere, which, when interacting with a force, has a certain physical characteristic (electrical, magnetic) or gravitational) material body creates a dynamic response of the parameters of this body, which is recorded in the experiment as a real flow of energy corresponding physical nature. Based on the definition, the force field represents a region of space, at each point of which the corresponding force interactions are determined.

\section{Finite element analysis}

There are four types of fundamental interactions, and, consequently, four types of force fields: Gravitational, weak interactions, electromagnetic fields of strong (nuclear) interactions.

Magnetic force: $\bar{F}_{\text {mag }}=q\left[\begin{array}{c}-- \\ v B\end{array}\right]$, electric field vectors $\bar{E}$ and magnetic induction $\bar{B}$ are components of a single electromagnetic field, that is, the electromagnetic field is a twocomponent $\left\{\begin{array}{c}-\bar{E} \\ B\end{array}\right\}$. The relationship between the components of the electromagnetic field: $\bar{B}=\frac{1}{c^{2}}\left[\begin{array}{c}- \\ v\end{array}\right]$

where $\quad c=3 \cdot 10^{8} \mathrm{M} / \mathrm{s}_{\text {- the speed of light; }} \boldsymbol{V}_{\text {- The speed of the charge. }}$

At the electromagnetic field on moving with speed

$v$ charge acting electromagnetic

force: $\bar{F}_{e m}=\bar{F}_{e l}+\bar{F}_{m a g}=q \bar{E}+q\left[\begin{array}{c}- \\ v \\ B\end{array}\right]$.

The combination of gravitational, electric, magnetic, electromagnetic fields is a single spatial field. From the side of a single spatial field, a force acts [13-14]: 


$$
\bar{F}=\bar{F}_{\text {grav }}+\bar{F}_{\text {el }}+\bar{F}_{\text {mag }}+\bar{F}_{\text {elmag }} \text {. }
$$

The general formula of a single field of force interaction of bodies in space can be written in the form:

$$
\begin{aligned}
& \bar{F}(r)=A \cdot \frac{\hbar c}{r^{2}} r=-\operatorname{grad}, \bar{F}(r)=\left(A \cdot \frac{\hbar c}{r^{2}} r\right)=-\operatorname{grad} U(r) \\
& \quad \hbar=\frac{\hbar c}{2 \pi} \text { - modified Planck's constant, } \quad c=\frac{1}{\sqrt{\varepsilon_{0} \mu_{0}}} \text { - the speed of light in a vacuum, }
\end{aligned}
$$

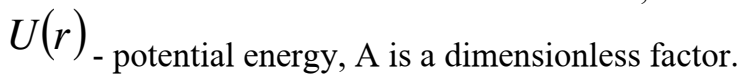

is determined by the products of the local physical parameters of two fixed interacting point bodies (electrically $q^{e}$, magnetic $q^{m}$ charges, m- masses), normalized to the square of the same dimension of the Planck constant $q_{p l}^{e}=\sqrt{4 \pi \varepsilon_{0} \hbar c}, q_{p l}^{m}=\sqrt{4 \pi \mu_{0} \hbar c}, m_{p l}=\sqrt{4 \pi \gamma_{0} \hbar c}$, composed of a combination of other fundamental physical constants).

According to, equations of systems of differential equations of a single force field of polarization of a physical vacuum with vector components of a polarization field strength $\bar{E}^{\text {pol }}(r)$ and vector potential fields ${ }^{-W}(r)$

a) $\operatorname{rot} \bar{E}^{p o l}=-\sqrt{\varepsilon_{0} \mu_{0}} \frac{\partial^{2} \bar{A}^{W}}{\partial t^{2}}$

b) $\operatorname{div}\left(\sqrt{\varepsilon_{0} \mu_{0}} \bar{E}^{p o l}\right)=\mathrm{O}$

c) $\operatorname{rot} \bar{A}^{W}=\sqrt{\varepsilon_{0} \mu_{0}} \bar{E}^{p o l}$

d) $\operatorname{div}\left(\sqrt{\varepsilon_{0} \mu_{0}} \bar{A}^{W}\right)=0$

These equations are nothing but the differential equations of the polarization field of the physical vacuum.

$$
\text { Wave equation for } \bar{E}^{\text {pol }}
$$

$\operatorname{rotrot} \stackrel{-p o l}{E}^{\text {poraddiv }} \bar{E}^{\text {pol }}-\Delta \bar{E}^{\text {pol }}=-\sqrt{\varepsilon_{0} \mu_{0}} \frac{\partial^{2}}{\partial t^{2}} \operatorname{rot} \bar{A}^{W}=$

$$
=-\sqrt{\varepsilon_{0} \mu_{0}} \frac{\partial^{2} E^{-}}{\partial t^{2}}=-\frac{1}{c_{w}^{2}} \frac{\partial^{2} \bar{E}^{-}}{\partial t^{2}}
$$


$\operatorname{rotrot} \bar{A}^{w}=\operatorname{graddiv} \bar{A}^{w}-\Delta \bar{A}^{w}=-\sqrt{\varepsilon_{0} \mu_{0}} \operatorname{rot} \stackrel{-}{E}^{\text {pol }}=$

$$
=-\varepsilon_{0} \mu_{0} \frac{\partial^{2} A^{w}}{\partial t^{2}}=-\frac{1}{c_{w}^{2}} \frac{\partial^{2} A^{w}}{\partial t^{2}}
$$

where $A^{w}$ - the vector potential of the force field of vacuum polarization from the point

of view of the field vector flux by induction, $\sqrt{\varepsilon_{0} \mu_{0}}$ - the speed of propagation of waves of polarization of the physical vacuum. Wave equations for polarization field components:

$\Delta \bar{E}-\frac{1}{c_{w}^{2}} \frac{\partial^{2} E^{-}}{\partial t^{2}}=0 \quad \Delta \bar{A}^{w}-\frac{1}{c_{w}^{2}} \frac{\partial^{2} A^{w}}{\partial t^{2}}=0$

where

$$
C_{w}=\frac{1}{\sqrt{\varepsilon_{0} \mu_{0}}}=c=3 \cdot 10^{8} \mathrm{M} / \mathrm{s} \text {. }
$$

These equations represent the equations of a single spatial field.

\section{Conclusion}

Based on the analysis of the physical characteristics of the forces of spatial interaction, the objectivity of the existence in nature of a single field of force interaction of bodies in the physical vacuum space due to the polarization of the vacuum medium in the presence of matter in it has been established.

It has been established that polarization waves transporting the energy of its excitation in the space of the vacuum sphere, which, when interacting with a certain physical characteristic (electrical magnetic or gravitational) of a material body, creates a dynamic response of the parameters of this body as a real energy flow. A method of representing a single spatial field with a complete system of differential and wave equations is shown.

\section{References}

1. A.A. Khalikov, O.A. Ibragimova. Scientific Almanac. 29,3-329:230-235(2017). https://ukonf.com/doc/na.2017.03.03.pdf

2. I.K. Kolesnikov, A.A. Khalikov, J.F. Kurbanov, O.A. Ibragimova, Problems of Energy and resource conservation. 1,2:204-220(2014).

3. A.A. Khalikov, I.K. Kolesnikov, J.F. Kurbanov, O.A. Research and development of a single spatial electromagnetic field and devices based on them. (2019).p.250.

4. V.V. Sidorenko. Irreversible processes in nature and technology. III (2011).p.215-219

5. A.A. Khalikov, I.K. Kolesnikov, J.F.Kurbanov. Fan wa technology to find out. (2019).p. 250

6. A.A. Khalikov, O.A. Ibragimova. IIUM Ingenering. 14,2:163-172(2013).

7. A.A. Khalikov, O.A. Ibragimova. VESTNIK. 1,29:63-69(2014).

8. A.A. Khalikov, I.K. Kolesnikov, J.F. Kurbanov, O.A. Ibragimova. The magazine "Problems of Energy and Resource Saving. 1,2:204-208(2014).

9. A.A. Khalikov, O.A. Ibragimova, I.K. Kolesnikov. Magazine "Energy saving and water treatment". Ed .: ENIV. 89,3:9-13(2014). 
10. A.A. Khalikov, O.A. Ibragimova. Center for Scientific Publications. III. (2015).p.2125.

11. A.A. Khalikov, O.A. Ibragimova. UNIVERSUM. 11:68(2019). https://7universum.com/ru/tech/archive/item/8231

12. A.A. Khalikov, I.K. Kolesnikov, S.S. Khalikov. The magazine "Problems of Energy and Resource Saving. 1,2:194-198(2015).

13. A.A. Khalikov, O.A. Ibragimova. "Energy saving and water treatment". ENIV. 2,106):14-18(2017).

14. A.A. Khalikov, O.A. Ibragimova, K.A. Musamedova. Science in the modern world. 9:28-31(2017). 